\title{
Use of electronic pharmacy transaction data and website development to assess antibiotic use in nursing homes
}

Sunah Song ${ }^{1,2,3}$, Brigid M. Wilson ${ }^{4,5}$, Joseph Marek ${ }^{6}$ and Robin L. P. Jump $3,4,5^{*}$ (D)

\begin{abstract}
Background: In 2017, the Centers for Medicare and Medicaid Services required all long-term care facilities, including nursing homes, to have an antibiotic stewardship program. Many nursing homes lack the resources, expertise, or infrastructure to track and analyze antibiotic use measures. Here, we demonstrate that pharmacy invoices are a viable source of data to track and report antibiotic use in nursing homes.

Methods: The dispensing pharmacy working with several nursing homes in the same healthcare corporation provided pharmacy invoices from 2014 to 2016 as files formatted as comma separated values. We aggregated these files by aligning elements into a consistent set of variables and assessed the completeness of data from each nursing home over time. Data cleaning involved removing rows that did not describe systemic medications, de-duplication, consolidating prescription refills, and removing prescriptions for insulin and opioids, which are medications that were not administered at a regular dose or schedule. After merging this cleaned invoice data to nursing home census data including bed days of care and publicly available data characterizing bed allocation for each nursing home, we used the resulting database to describe several antibiotic use metrics and generated an interactive website to permit further analysis.
\end{abstract}

Results: The resultant database permitted assessment of the following antibiotic use metrics: days of antibiotic therapy, length of antibiotic therapy, rate of antibiotic starts, and the antibiotic spectrum index. Further, we created a template for summarizing data within a facility and comparing across facilities. https://sunahsong.shinyapps.io/USNur singHomes/.

Conclusions: Lack of resources and infrastructure contributes to challenges facing nursing homes as they develop antibiotic stewardship programs. Our experience with using pharmacy invoice data may serve as a useful approach for nursing homes to track and report antibiotic use.

Keywords: Nursing home, Antimicrobial stewardship, Antibiotic stewardship, Fluoroquinolones

\section{Background}

In the United States, nursing homes care for approximately 4 million individuals annually [1]. Antibiotics are some of the most frequently prescribed medications in

*Correspondence: robin.jump@va.gov; robinjump@gmail.com

${ }^{3}$ Department of Population and Quantitative Health Sciences, School

of Medicine, Case Western Reserve University, Cleveland, OH, USA

Full list of author information is available at the end of the article nursing homes, with point prevalence studies indicating that on any given day, approximately $10 \%$ of nursing home residents receive antibiotics [2-5]. Unfortunately, $25-75 \%$ of antibiotic use in nursing homes is inappropriate [6-10]. Reasons to deem an antibiotic as inappropriate include an unnecessary indication (i.e., a viral infection), excessive length of therapy, selecting the wrong dose, or choosing an agent that is unnecessarily 
broad or does not treat the suspected bacterial pathogen. Systemic antibiotics may lead to adverse events including side effects, allergic reactions, drug-drug interactions, Clostridioides difficile infection (CDI), and selection for multi-drug resistant organisms (MDROs).

Antibiotic stewardship seeks to reduce inappropriate antibiotic use, which in turn supports patient safety and quality healthcare. Driven by the increasing prevalence of both CDI and MDROs across all healthcare settings $[11,12]$, the Centers for Medicare and Medicaid Services (CMS) required all nursing homes to have an antibiotic stewardship program by November 2017 [13]. In support of these efforts, the Centers of Disease Control and Prevention (CDC) developed a framework for antibiotic stewardship implementation called the Core Elements that detail actions critical for the success of antibiotic stewardship implementation [14]. Two of the seven Core Elements important to successful antibiotic stewardship programs are tracking and reporting antibiotic use. Many nursing homes lack resources, expertise, and infrastructure to assess antibiotic use in their buildings. Previous work has addressed the utility of using data from pharmacy invoices to measure antibiotic use in nursing homes [15]. Expanding on those efforts, we recently used pharmacy invoice data to characterize antibiotic use in 29 nursing homes in the United States [16]. Here, we describe in detail the process of transforming pharmacy invoices into a robust dataset that permits assessment of the following important antibiotic use metrics: overall days of therapy; length of therapy; antibiotic starts; use of intravenous agents; spectrum of antibiotic use based on specific antibiotic classes. We also developed a web interface that supports further analysis as well as tracking and reporting antibiotic use.

\section{Methods}

\section{Data sources}

The primary source of data was pharmacy invoices issued from a single dispensing pharmacy to several nursing homes within the same healthcare corporation. As previously described, invoice data accounts for medications requested by the nursing home for residents receiving skilled nursing care, for whom CMS is primary insurance provider [16]. We also obtained pharmacy dispensing records 6 nursing homes for data validation. These data indicate the medications dispensed from the pharmacy to the nursing home for residents receiving skilled nursing care (short-stay) and custodial or residential care (longstay). The healthcare corporation provided monthly census data, indicating the bed-days of care, for individual nursing homes. Data describing nursing home characteristics came from the Long-Term Care: Facts on Care in the US (LTCFocus) websites and from Nursing Home
Compare, both of which use data from CMS $[17,18]$. The Institutional Review Board (IRB) at the VA Northeast Ohio Healthcare System approved the study protocol.

\section{Data aggregation}

Transforming pharmacy invoices into a database suitable for analysis was a multistep process (Fig. 1). The healthcare corporation sent comma separated values (CSV)

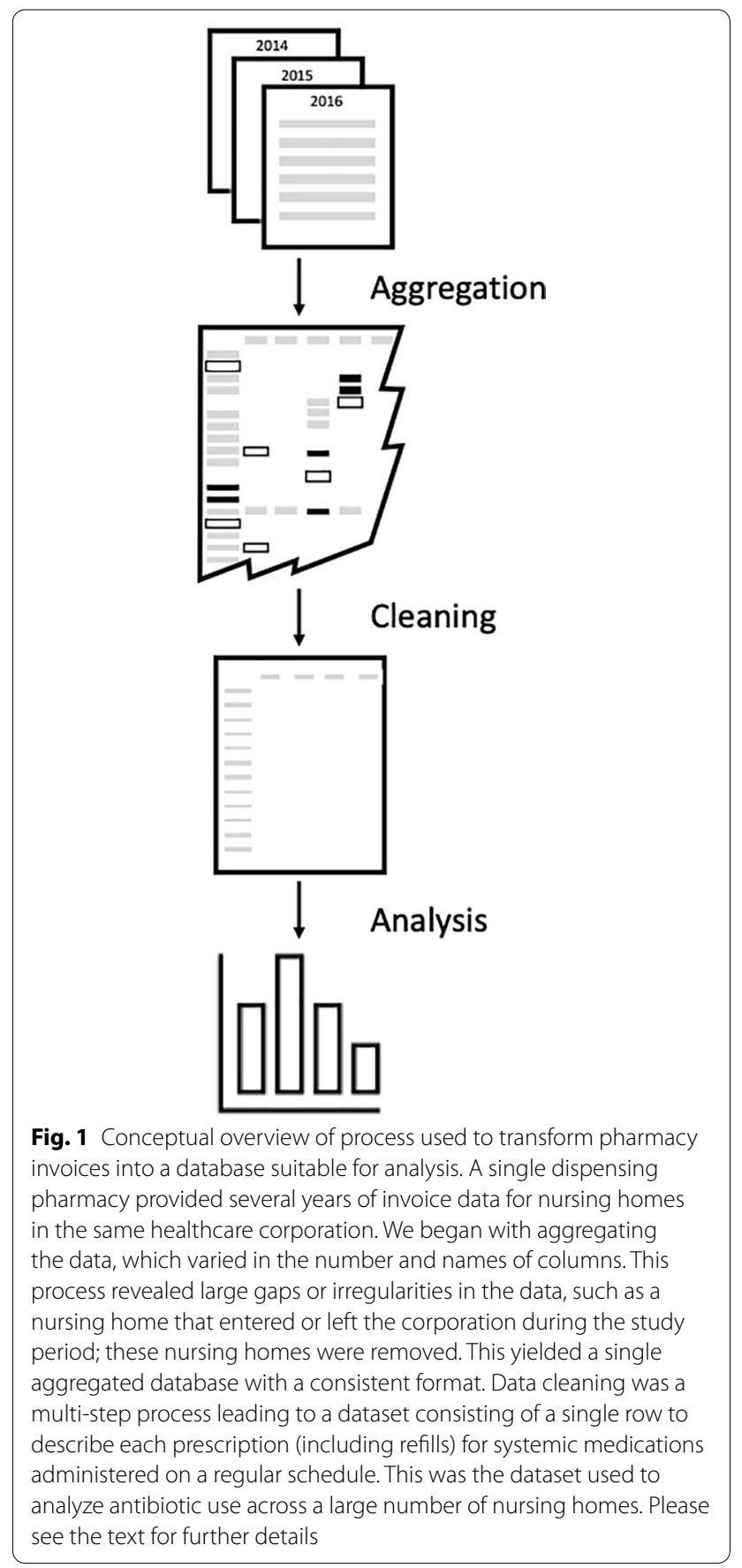


files containing invoices from January 2014 to June 2018. Each file included monthly invoices from several nursing homes, with each row in the file representing a transaction related to a prescription for an individual resident. We aggregated these files, which varied in the number of columns, into a single database with uniform column names and standardized data formats within each column. These data were then assessed for their distribution over time, by monthly intervals, as well as by individual nursing homes. Gaps or notable changes in the number of invoices from individual nursing homes were discussed with leadership at the healthcare corporation who provided context for some of these changes, such as a temporary closure or change in the resident population.

\section{Data cleaning}

Data cleaning involved the following steps in sequence: removing rows with incomplete data, removing invoices for non-pharmaceutical items, removing lines indicating reimbursement, de-duplication, consolidating prescription refills into a single course, removing non-systemic medications, and removing insulin and opioids as administration of these medications often includes fluctuations in the dose or schedule. A clinician (RJ) and data scientist $(\mathrm{BW})$ reviewed both the removed and retained data at each step to confirm it was clinically reasonable and accurate.

First, we removed rows that were missing any elements from the following essential columns: Facility Name, Transaction Date, Prescription Number, Drug Name, Days Supply, Amount, Route of Administration (i.e., oral or intravenous), Physician, and Health Care Payor. Second, we removed all rows pertaining to invoices for non-pharmaceutical agents (e.g., kits for placing intravenous lines or normal saline). Third, rows for reimbursement or payment, indicated by negative monetary values, were removed. Fourth, we reconciled records for which we found multiple rows with the same prescription number, medication, and transaction date according to the logic below for the two scenarios observed:

- Case One If there was more than one row with the same prescription number, medication, transaction date, physician, and days supply, then we retained only one row.

- Case Two If there was more than one row with the same prescription number, medication, transaction date, physician and different days supply, we retained the row with the greatest number of days supply.

Fifth, we addressed instances of more than one row with the same prescription number and medication but with different transaction dates, with later transaction dates representing medication dispensing events or refills. To consolidate multiple rows with the same prescription number, we added up the total days supply across rows and considered this to be the length of therapy. Sixth, we used several regular expressions to search for and remove rows for non-systemic medications, i.e., medications administered topically, as otic or ophthalmologic drops, as suppositories, or via inhalation (Table 1). This left rows for medications administered through oral, intravenous, and intramuscular routes. Finally, we used regular expressions specific to insulin and opioids to remove rows for these medications, which are frequently administered using a variable dose and schedule.

Table 1 Regular expressions used to remove non-systemic medications

\begin{tabular}{|c|c|c|}
\hline Regular expressions & Description and example & Strings \\
\hline$[\wedge[:$ alpha: $]]$ STRING & $\begin{array}{l}\text { Drug Name that ends with [Non Alphabetic } \\
\text { Character] STRING } \\
\text { e.g., HYDROCORTISONE 2.5\% CREAM }\end{array}$ & $\begin{array}{l}\text { CREAM, CRM, DROP, EAR, EYE, EYEOINT, FOAM, } \\
\text { GEL, INHALER, INHAL, LOTION, MOUTHWAS, } \\
\text { MOUTHWASH, NASAL, OINT, OINTM, OINT- } \\
\text { MENT, OPTH, OPHTH, OTIC, PATCH, RINSE, } \\
\text { SPRAY, TOPIC, TOPICAL, SHAMP, SHAMPOO, } \\
\text { RINGERS, 1:1 }\end{array}$ \\
\hline$[\wedge[$ :alpha: $]]$ STRING [^[:alpha: $]]$ & $\begin{array}{l}\text { Drug Name contains [Non Alphabetic } \\
\text { Character] STRING [Non Alphabetic Char- } \\
\text { acter] } \\
\text { e.g., PERMETHRIN CREAM 5\% 60GM }\end{array}$ & $\begin{array}{l}\text { CREAM, CRM, DROP, EAR, EYE, EYEOINT, FOAM, } \\
\text { GEL, INHALER, INHAL, LOTION, MOUTHWAS, } \\
\text { MOUTHWASH, NASAL, OINT, OINTM, OINT- } \\
\text { MENT, OPTH, OPHTH, OTIC, PATCH, RINSE, } \\
\text { SPRAY, TOPIC, TOPICAL, SHAMP, SHAMPOO, } \\
\text { RINGERS, } 1: 1\end{array}$ \\
\hline ^STRING [^[:alpha: ] ] & $\begin{array}{l}\text { Drug Name that starts with STRING [ Non Alpha- } \\
\text { betic Character ] } \\
\text { e.g., GELFOAM SIZE } 50 \text { SPONGE }\end{array}$ & GELFOAM, WATER INJ, SSD, ABH \\
\hline$\wedge$ STRING $[\wedge[$ :blank: $]] \% *$ & $\begin{array}{l}\text { Drug Name that starts with STRING [ blank ] and zero } \\
\text { or more times of \% } \\
\text { e.g., LIDOCAINE HCL 2\% JEL }\end{array}$ & DS, LIDOCAINE, NACL, SODIUM CHL \\
\hline
\end{tabular}




\section{Data analysis}

From the resulting set of systemic medications, we identified antibiotics, categorizing these into several subclasses, as well as anti-hypertensives from several subclasses (beta-blockers, angiotensin-converting enzyme inhibitors [ACE-Is] and angiotensin 2 receptor blockers [ARBs]) (Additional file 1: Table S1). The rational for using antihypertensives is that these agents are commonly prescribed, including among nursing home residents, and have not been subject to recent changes in guidance or recommendations that might lead to changes in their use. In combination with census data, we quantified systemic medications by the number of starts per 1000 bed days of care (BDOC), days of therapy (DOT) per 1000 BDOC, and length of therapy (in days). Both the number of starts/1000 BDOC and DOT/1000 BDOC are common metrics to assess antibiotic use $[19,20]$. The number of starts/1000 BDOC assesses the frequency of prescribing. DOT/1000 BDOC is the number of calendar days a medication is administered standardized to total days in care and describes the overall rate of use; we determined this based on the days supply [21, 22]. For some medications, particularly those with several refills or with a transaction date near the end of a month, the total DOT exceeded the calendar days in that month. Accordingly, for calculating DOT/1000 BDOC at monthly intervals, we distributed the DOT across months. For example, if an anti-hypertensive was prescribed for 90 days starting December 3, 2014, the DOT would be allocated as follows: 28 DOT for December 2014, 31 DOT for January 2015, 28 DOT for February 2015 and 3 DOT for March 2015 for a total of 90 DOT.

We also used previously described antibiotic spectrum index (ASI) to summarize prescribed antibiotics on a quantitative scale from 1 to 13, with lower values indicating more narrow-spectrum agents and higher values more broad-spectrum agents (Additional file 1: Table S2) [23]. We calculated the monthly mean ASI among the antibiotic DOT for each facility.

\section{Data validation}

To validate our analysis dataset, we used dispensing data from 2016 for six of the nursing homes. These data were cleaned and analyzed using the same process described for the larger invoice dataset. We used the Mann-Kendall trend test and Spearman rank correlation test to compare antibiotic DOT/1000 BDOC from the invoice and dispensing datasets.

\section{Website development}

Using an open source Shiny R package, we built an interactive web application to show the result of each steps for data cleaning and further antibiotic use analysis [24].
The shinyapps.io cloud hosting allowed us to deploy the application online in secure environment. The application runs in its own protected domain on the cloud server and user access is always secure sockets layer (SSL) encrypted.

\section{Results \\ Data aggregation}

The dispensing pharmacy provided a total of 51 files with 15 to 27 columns describing transactions from $1 / 1 / 2014$ through 6/30/2018. We used column headers and nursing homes names to reconcile the meta-data, generating a file with 28 columns and nearly 2 million rows including 82 distinct nursing home names. Only 11 columns were retained for the aggregation; of the discarded columns, 11 of the columns had $>40 \%$ missing data and six additional columns had $>90 \%$ missing data (Table 2 ).

Among the 82 distinct nursing home names, we excluded 37 nursing home names that contributed $\leq 12$ months of data to the study period $(1 / 1 / 2014$ 12/31/2016). We reviewed the remaining 45 nursing homes names and noted that some were very similar. In consultation with leadership from the healthcare corporation, we consolidated similar names. For example, we assigned 'SleepyHollow' as a facility name for rows that contained 'SleepyHollow', 'Sleepy Hollow', or 'Sleep Hollow CCRC'; CCRC is an acronym for continuing care retirement community. This resulted in 32 distinct nursing home names.

For each nursing home, we assessed the number of rows attributed to each month from 2014 to 2016 . We found that three nursing homes had a $>20 \%$ change in the number of rows over three consecutive months. Discussion with leadership from the healthcare corporation revealed that one building had closed and that two had changed the population served sufficiently to alter the volume of pharmacy invoices (i.e., from those in need of skilled nursing care to those with psychiatric illness). Removing these facilities from the list resulted in a database with ten essential columns and 995,785 rows across 29 nursing homes.

\section{Data cleaning}

The primary aim of data cleaning was to create a database with one row per prescription that included complete data including the prescribed drug, the date of the prescription, the duration of the prescription, the location (nursing home) of the prescription, and the prescribing provider. To achieve this aim, we took the following steps that generally consisted of excluding, aggregating, or reconciling rows within our starting database.

From the aggregated database, only 18,525 (1.8\%) of rows had data missing from the ten columns deemed 
Table 2 Column headings used to aggregate files

\begin{tabular}{|c|c|c|}
\hline Final column name & Original Column Name(s) & Description of data elements \\
\hline Facility name & Facility name & 82 distinct expressions for names of nursing homes \\
\hline Medication & Drug name, medication name, agent, description & 13,549 distinct medications \\
\hline Dose & Dose, quantity, qty & $\begin{array}{l}\text { Quantities in grams or milligrams for oral medications and in } \mathrm{gm} / \mathrm{mL} \text { for } \\
\text { intravenous medications }\end{array}$ \\
\hline Days supply & Days supply, days of supply, days dispensed & Ranged from 0 to 946 days \\
\hline Prescription number & Prescription number, Rx & 938,007 distinct numbers \\
\hline Transaction date & Transaction date & Ranged from $9 / 23 / 2007$ to $6 / 25 / 2018$ \\
\hline Amount & Amount & Ranged from $\$ 0$ to $\$ 15,000.08$ \\
\hline Route of administration & Route of administration, inventory category & 40 distinct expressions \\
\hline Physician & Physician & 7971 distinct physicians \\
\hline Health care payor & Payor, pay type description & $\begin{array}{l}13 \text { distinct descriptions reflecting the entity, typically an insurance } \\
\text { company, that ultimately pays for the medication, listed as general } \\
\text { categories of Medicare, Medicaid, the nursing home, Veterans Affairs, } \\
\text { hospice, and private parties }\end{array}$ \\
\hline National drug code & NDC & $\begin{array}{l}\text { National Drug Code, } 7 \% \text { missingness, } 9885 \text { distinct codes. Ultimately, not } \\
\text { used in data analysis }\end{array}$ \\
\hline
\end{tabular}

essential for analysis; these were removed (Table 3). Rows describing non-pharmaceutical items and records indicating reimbursement were also removed, leaving 648,326 rows. When we considered the two different cases of duplications, Case One (same prescription number, medication, transaction date, physician, and days supply) accounted for 48,088 rows removed and Case Two (same prescription number, medication, transaction date, physician, and different days supply) accounted for 10,663 rows removed.

Of the remaining 589,575 lines of data, there were 136,172 rows that did not have a unique prescription number. Rows with the same prescription number indicated a dispensing event from the pharmacy of the same medication at the same dose to the same person. Most often, these were refills for medications used to treat chronic conditions, such as anti-hypertensives for high blood pressure. In other instances, these represented several dispensing events for a limited number of doses of a medication with a limited shelf-life, most often intravenous antibiotics. For both situations, we added up the total days supply among rows with the same prescription number.

In the final step of data cleaning, we used regular expressions to remove rows for non-systemic medications (35,899 rows) and rows for medications administered without a fixed dose or schedule, specifically insulin and opioids $(49,208$ rows). The clean database had 368,296 rows, which was $37 \%$ of the those present in the aggregated dataset.

As previously described, we used the clean database to describe antibiotic use across 29 US nursing homes [16], which included steps that allowed us to summarize antibiotic use at yearly intervals based on the date and duration of a prescription. Allocating the DOT across calendar months indicated that $54 \%$ of prescriptions carried over to more than 1 month and 6\% extended for more 2 months or longer. Further analysis was performed after merging nursing home census data (e.g., bed days of care, occupancy rate) to the cleaned antibiotic use

Table 3 Rows removed when cleaning aggregated dataset

\begin{tabular}{|c|c|c|c|}
\hline Step & Description & Rows removed & Rows remaining (\%) \\
\hline$(\mathrm{n} / \mathrm{a})$ & Records in the aggregated dataset & & 995,785 \\
\hline 1 & Removed rows missing data from essential columns & 18,525 & $977,260(98 \%)$ \\
\hline 2 & Removed rows for non-pharmaceuticals & 99,362 & $877,898(88 \%)$ \\
\hline 3 & Removed rows indicating reimbursements & 229,572 & $648,326(65 \%)$ \\
\hline 4 & Removed duplicate rows & 58,751 & $589,575(59 \%)$ \\
\hline 5 & Removed rows indicating medication refills & 136,172 & $453,403(46 \%)$ \\
\hline 6 & Removed rows for non-systemic medications & 35,899 & $417,504(42 \%)$ \\
\hline 7 & Removed rows for insulin and opioids & 49,208 & $368,296(37 \%)$ \\
\hline
\end{tabular}


data to standardize antibiotic use to bed days of care and analyze as rates. We further merged data elements used to describe operational characteristics, resident demographics, and quality indicators specific to nursing homes $[17,18]$. In summary, our results indicated the following: between 2014 and 2016, the overall rate of antibiotic use did not change; fluoroquinolones were the most commonly prescribed class of antibiotics; and the mean ASI was similar across nursing homes [16]. When assessed based on the number of beds, we did not observe notable differences in rates of total antibiotic use among nursing homes with $<100$ beds compared to those with 100-150 and those with $>150$ beds.

\section{Data validation}

We validated the antibiotic use data derived from invoice data with dispensing data obtained for six nursing homes. Antibiotic DOT/1000 BDOC based on dispensing data was consistently greater than that those based on invoice data (Additional file 1: Figure S1). This is consistent with the resident populations encompassed by these datasets: dispensing data accounts for all residents in the nursing home while invoice data accounts primarily for residents receiving skilled nursing care. Comparing the monthly 2016 measures from both data sources, the Spearman rho correlation for antibiotic DOT/1000 BDOC was high $(>0.7)$ for two nursing homes and moderate $(0.5-0.7)$ for three nursing homes. When we focused on intravenous antibiotics, typically administered only to residents receiving skilled nursing care and thus expected to occur in invoice data with a CMS payor, the Spearman rho correlation values increased for five of the six nursing homes. For one of the nursing homes, the correlation decreased from 0.81 to 0.39 . Leadership from the healthcare corporation confirmed that the operational characteristics for this nursing home were similar to the others and suggested the possibility of errors related to transfer of data specific to compounded intravenous medications, which are often handled through a different software system.

\section{Website}

Figure 2 shows the interface, which included an array information organized into five tabs. The first tab, Number of Records, provided an overview of the number of records removed with each cleaning step. The second tab,

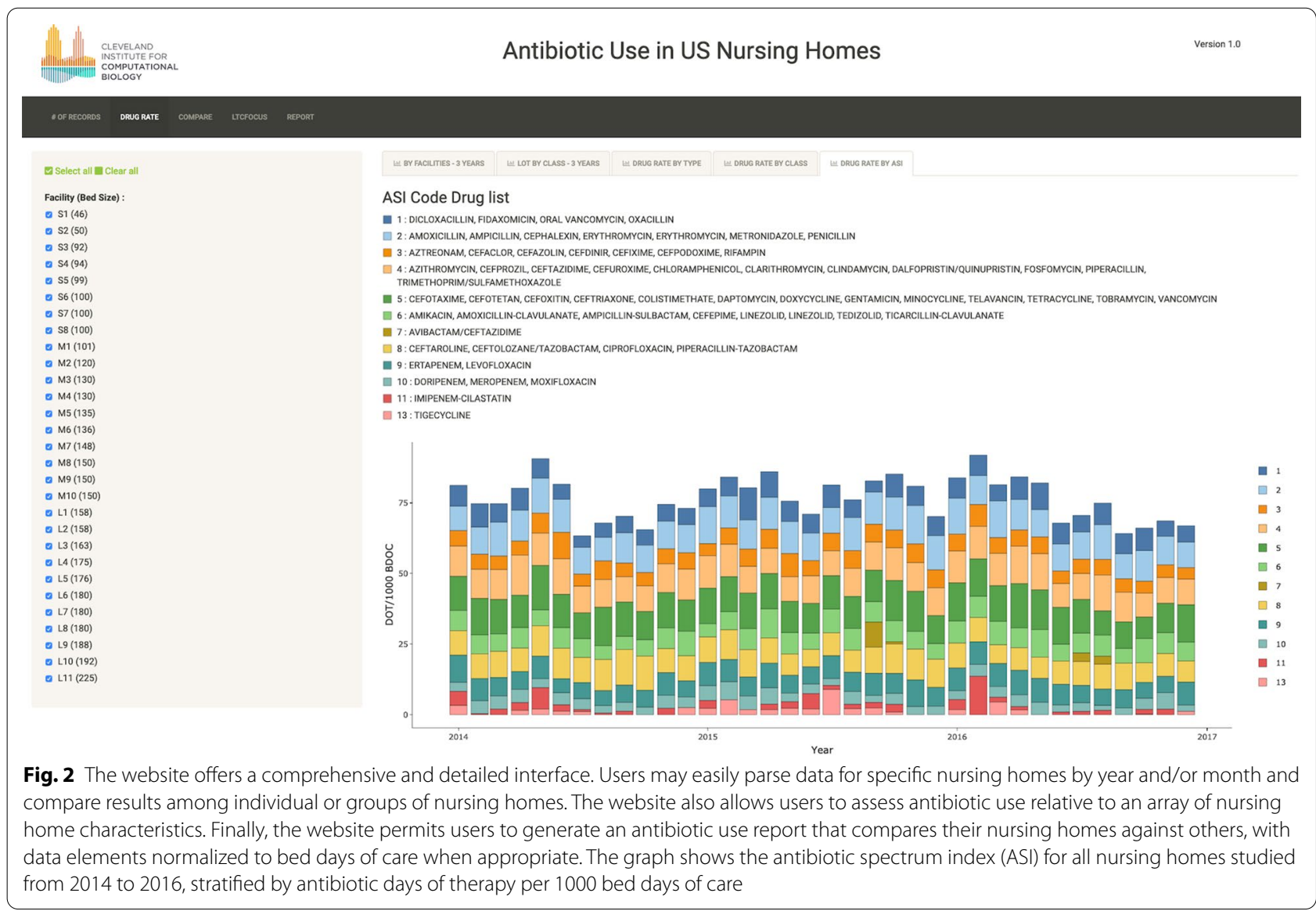


Drug Rates, had five sub-tabs that gave access to several antibiotic use metrics including overall rates of antibiotic use, length of antibiotic therapy, antibiotic use categorized by class of agent, and the antibiotic spectrum index (shown in the graph in Fig. 2), which provides a quantitative comparison of the activity of antibiotics against bacterial pathogens. An additional sub-tab compared antibiotic use to all systemic medications and to use of anti-hypertensive agents. With the third tab, Compare, users could parse nursing homes into two subgroups and use robust data visualization to compare several antibiotic use metrics. The fourth tab, LTCFocus, provided data and graphs that describe relationships between antibiotic use and nursing home characteristics, as assessed by CMS and made available through LTCFocus.org [17]. Finally, the Report tab generated two simple graphs that provide comparative feedback so that both clinical and administrative staff can understand how antibiotic use at their facility compares to antibiotic use at nursing homes with a similar number of beds (Fig. 3). Filters available within the website permitted users to examine individual or groups of nursing homes and to specify the time frame assessed. Another feature, labeled Data Table and at the bottom on most of the tabs, permitted users to download data for further analysis. Across all the tabs, tooltips are also available when the user hovers over, focuses on, or touches an element of figures. Additional details of results are available in the shiny repository, https://sunah song.shinyapps.io/USNursingHomes/.

\section{Discussion}

Our intent was to provide a blueprint to support nursing homes in using an existing dataset, pharmacy invoices, to construct a dataset that permits tracking and reporting antibiotic use. Analyzing the data derived from the cleaning approach described here, we observed a frequency of fluoroquinolone use as well as steady rates of antibiotic use between 2014 and 2016 that were consistent with

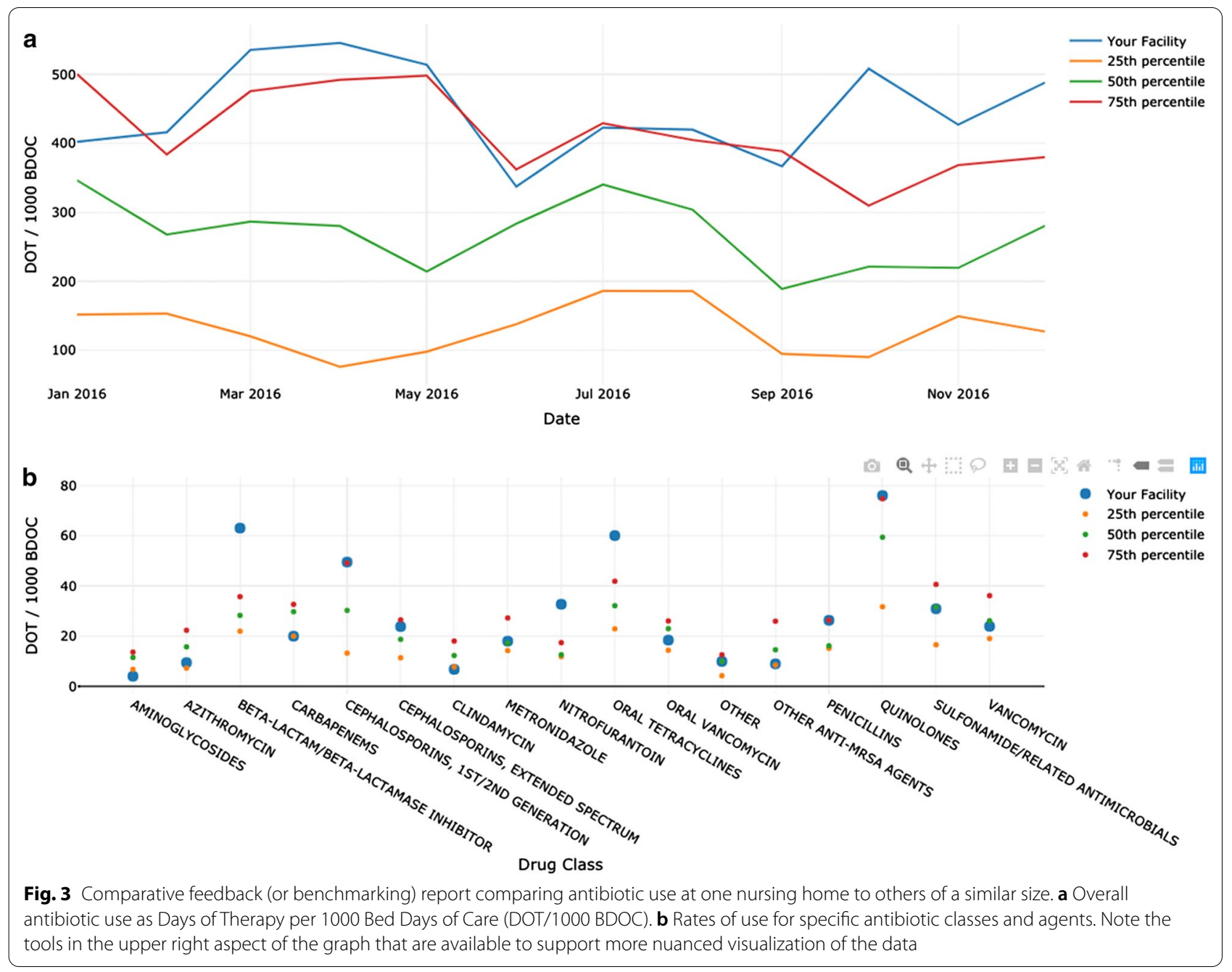


other reports that use clinical or surveillance datasets to assess antibiotic use in US nursing homes $[5,15,25,26]$. Validation of a subset of the data derived from invoice data using pharmacy dispensing data found similar overall trends for total antibiotic use and for intravenous antibiotic use in five of the six nursing homes assessed. These coupled results support the use of cleaned invoice data as a valid measure of antibiotic use. 4/28/2021 4:23:00 PMFurthermore, we developed a website specifically designed to serve as an interface that ready visualization of several antibiotic use metrics. The approach described and the resulting website support comparing antibiotic use among nursing homes within a network or region served by the same dispensing pharmacy.

The strengths of the website are the displays that permit visualization of changes over time as well as comparisons to other sites. The user-friendly design encourages changing views to answer questions specific to an individual site. Furthermore, while tracking and reporting antibiotic use typically falls under the purview of a nursing home's infection preventionist(s), the members of the antibiotic stewardship team or the Quality Assurance and Performance Improvement (QAPI) committee could also use the website. They may be interested in assessing changes following an intervention, such as restricting use of fluoroquinolones, the most commonly prescribed antibiotic class, to residents with severe penicillin allergies. Examination of antibiotic use over time makes it possible to detect unintended consequences of changes. Building on the previous example, restricting use of fluroquinolones may lead to an increase in other antibiotics, such as clindamycin or doxycycline. Inclusion of identifying information may enable assessment of additional antibiotic stewardship metrics, such as conversion from intravenous to oral antibiotic administration.

While the steps of data aggregation and cleaning described above are applicable to invoice formats present in the administration of most nursing homes, the expertise needed to transform pharmacy invoice data into a clean database, and to maintain those processes, may be available only to nursing homes that are part of a larger healthcare system. Guidelines for hospitalbased antibiotic stewardship programs call for a core multidisciplinary antimicrobial stewardship team that includes an infectious diseases-trained physician and clinical pharmacist as well as a clinical microbiologist, hospital epidemiologist, information specialist, and an infection preventionist [27]. Except for an infection preventionist, individual nursing homes do not typically employ individuals with these skill sets. Indeed, CMS indicates that in nursing homes, the antibiotic stewardship program is part of the broader infection prevention and control program, which is led by one or more infection preventionists [13]. CMS estimates that for the average nursing home, only $15 \%$ of a fulltime equivalent (FTE) is required for the entirety of the infection prevention and control program [13]. The disproportionate effect of the COVID-19 pandemic on nursing home residents, which account for only $4 \%$ of cases and over 33\% of deaths in the US [28], is a strong indicator more resources are needed to support infection prevention and control in nursing homes, which in turn will enhance resident safety. We suggest that $15 \%$ of an FTE may be an adequate allocation specifically for tracking and reporting antibiotic use in nursing homes. Additional effort from a clinical pharmacist or physician may be necessary to provide oversight and help with data interpretation.

Most hospitals use information from their electronic medical records to assess antibiotic use; this can include medication orders, dispensing events, and bar-coded medication administration (BCMA) data. Previous work at a Veterans Affairs hospital compared medical orders with BCMA data to assess antibiotic metrics. While both approaches had errors, BCMA data was more accurate, consistent with this being a process that confirms that the correct medication at the correct dose is being given to the correct patient at the point of care [29]. As assessment of BCMA data from 13 nursing homes, however, found an error rate of $90 \%$, with most errors related to the timing of medications that require administration (i.e., every 4 to $6 \mathrm{~h}$ ) [30]. While these findings belie the expectation of strong medication adherence in all institutionalized settings, using electronic medical record to assess antibiotic use in nursing homes would likely be superior to pharmacy invoice data [31]. Not all nursing homes have adopted electronic medical records and those that have may not have personnel with time and expertise to extract antibiotic use data for tracking and reporting purposes.

Invoice data serves as a proxy for antibiotic use in nursing homes, with limitations that include not capturing changes from intravenous to oral administration or accounting for discontinuation of antibiotics. Kabbani et al. also used invoice data to assess antibiotic use. In contrast to the dataset discussed here, their invoices did not have the prescription numbers that permitted us to link dispensing events related to a single medication. Even with this limitation, Kabbani et al. [15] were able to compare the antibiotic days of therapy among 12 nursing homes, finding a five-fold difference in rates between the lowest- and highest-use homes. The use of dispensing data would likely permit more accurate assessment of discontinued medications, which would help to improve 
the accuracy for antibiotic use metrics in intravenous agents in particular, as these may requiring multiple dispensing events for a single course. Dispensing data, however, may not be as readily accessed as invoice data.

Our approach has limitations. First, the website is designed to permit comparison of several affiliated nursing homes that are served by a single dispensing pharmacy. Independent nursing homes may still use the process outlined, along with the website, to assess antibiotic use over time. Second, the steps used to clean the data may not apply to data format used by some pharmacy invoice systems. We assert that the general approach outlined will serve as a template for information technologists to work directly with their own data. They may be able to use additional data fields, such as the indications for antibiotics prescribed, to yield further information relevant to antibiotic stewardship efforts. Third, like any system, errors may accrue. Minor discrepancies inherent to using pharmacy invoices include unbilled single-dose medication administration, cancelled orders, changes in treatment, or resident transfers. As long as these events remain at a reasonably low and fairly consistent level, they should not interfere with the overall meaning of antibiotic use data. More notable problems with the data are likely be noticed by end-users who will need to work with information technologists to identify potential sources of error and validate the data.

\section{Conclusions}

The process for transforming pharmacy invoices into antibiotic use data may help support overall antibiotic stewardship efforts for some nursing homes. Readily tracking and reporting antibiotic use should complement other efforts, such as those of nursing homes participating in the National Healthcare Safety Network (NHSN), which supports tracking urinary tract infections, C. difficile infections and multidrug-resistant organisms [32]. Ultimately, our hope is that this approach will reduce some of the barriers to tracking and reporting antibiotic use and will become another tool used by nursing homes to improve the use of antibiotics and thus, enhance the safety and quality of care for their residents.

\footnotetext{
Abbreviations

GRECC: Geriatric Research Education and Clinical Center; VA: Veterans Affairs; CDI: Clostridioides difficile Infection; MDRO: Multi-drug resistant organism; CMS: Centers for Medicare and Medicaid Services; CDC: Centers of Disease Control and Prevention; LTCFocus: Long-Term Care: Facts on Care in the US; CSV: Comma separated values; ACE-I: Angiotensin-converting enzyme inhibitor: ARB: Angiotensin 2 receptor blocker; DOT: Days of therapy; BDOC: Bed days of care; ASI: Antibiotic spectrum index; CCRC: Continuing care retirement community; US: United States; NHSN: National Healthcare Safety Network; IRB: Institutional Review Board.
}

\section{Supplementary Information}

The online version contains supplementary material available at https://doi. org/10.1186/s12911-021-01509-7.

Additional file 1: Table S1. Lists the class, subclass, and name of the antibiotics and anti-hypertensive agents evaluated. Table S2 details the Antibiotic Spectrum Index scores for individual antibiotics. Figure S1. Compares antibiotic use for six nursing homes based on data derived the pharmacy invoices and from dispensing data.

\section{Acknowledgements}

The findings and conclusions in this document are those of the authors, who are responsible for its content, and do not necessarily represent the views of the Veterans Administration or of the United States Government.

\section{Authors' contributions}

Study conception and design: RJ, SS, BW, JM. Data generation: SS, BW, JM. Data analysis and interpretation: SS, BW. Preparation and critical revision of the manuscript: SS, BW, JM, RJ. All authors read and approved the final manuscript.

\section{Funding}

This work was supported in part by a grant from Steris and by funds and facilities provided by the Cleveland Geriatric Research Education and Clinical Center (GRECC) at the VA Northeast Ohio Healthcare System. The funding bodies had no role in the design of the study and collection, analysis, and interpretation of data and in writing the manuscript.

\section{Availability of data and materials}

The datasets generated during the current study are available in the shiny repository, https://sunahsong.shinyapps.io/USNursingHomes/.

\section{Declarations}

\section{Ethics approval and consent to participate}

The healthcare corporation granted permission to access de-identified pharmacy invoice and dispensing data for the nursing homes included in this study.

\section{Consent for publication}

Not applicable.

\section{Competing interests}

The authors declare that they have no competing interests.

\section{Ethics approval}

The Institutional Review Board (IRB) at the VA Northeast Ohio Healthcare System approved the study protocol.

\section{Author details \\ ${ }^{1}$ Cleveland Institute for Computational Biology, Cleveland, OH, USA. ${ }^{2}$ Depart- ment of Computer and Data Sciences, School of Engineering, Case Western Reserve University, Cleveland, OH, USA. ${ }^{3}$ Department of Population and Quan- titative Health Sciences, School of Medicine, Case Western Reserve Univer- sity, Cleveland, OH, USA. ${ }^{4}$ Division of Infectious Diseases and HIV Medicine in the Department of Medicine, School of Medicine, Case Western Reserve University, Cleveland, OH, USA. ${ }^{5}$ Geriatric Research Education and Clinical Center (GRECC), Veterans Affairs (VA) Northeast Ohio Healthcare System, 10701 East Blvd., Cleveland, OH 44106, USA. ${ }^{6}$ CommuniCare Health Services, Cincinnati, $\mathrm{OH}, \mathrm{USA}$.}

Received: 20 January 2021 Accepted: 27 April 2021

Published online: 05 May 2021

\footnotetext{
References

1. American Health Care Association. Quality reports. https://www.ahcancal. org/qualityreport/Pages/default.aspx. Accessed 1 Aug 2019.
} 
2. D'Agata E, Mitchell SL. Patterns of antimicrobial use among nursing home residents with advanced dementia. Arch Intern Med. 2008;168:357-62.

3. Mitchell SL, Shaffer ML, Loeb MB, Givens JL, Habtemariam D, Kiely DK, et al. Infection management and multidrug-resistant organisms in nursing home residents with advanced dementia. JAMA Intern Med. 2014;174:1660-7.

4. Pakyz AL, Dwyer LL. Prevalence of antimicrobial use among United States nursing home residents: results from a national survey. Infect Control Hosp Epidemiol. 2010;31:661-2.

5. Thompson ND, LaPlace L, Epstein L, Thompson D, Dumyati G, Concannon C, et al. Prevalence of antimicrobial use and opportunities to improve prescribing practices in U.S. nursing homes. J Am Med Dir Assoc. 2016:17:1151-3.

6. Daneman N, Gruneir A, Bronskill SE, Newman A, Fischer HD, Rochon $\mathrm{PA}$, et al. Prolonged antibiotic treatment in long-term care: role of the prescriber. JAMA Intern Med. 2013;173:673-82.

7. Lim CJ, Kong DCM, Stuart RL. Reducing inappropriate antibiotic prescribing in the residential care setting: current perspectives. Clin Interv Aging. 2014;9:165-77.

8. Nicolle LE, Bentley DW, Garibaldi R, Neuhaus EG, Smith PW. Antimicrobial use in long-term-care facilities. Infect Control Hosp Epidemiol. 2000;21:537-45.

9. Peron EP, Hirsch AA, Jury LA, Jump RLP, Donskey CJ. Another setting for stewardship: high rate of unnecessary antimicrobial use in a veterans affairs long-term care facility. J Am Geriatr Soc. 2013;61:289-90.

10. Rotjanapan P, Dosa D, Thomas KS. Potentially inappropriate treatment of urinary tract infections in two rhode island nursing homes. Arch Intern Med. 2011;171:438-43.

11. Threat Report 2013 | Antimicrobial Resistance | CDC. http://www.cdc gov/drugresistance/threat-report-2013/index.html. Accessed 20 Oct 2013.

12. CDC. The biggest antibiotic-resistant threats in the U.S. Centers for Disease Control and Prevention. 2019. https://www.cdc.gov/drugresistance/ biggest-threats.html. Accessed 10 Mar 2020.

13. Medicare and Medicaid Programs; Reform of Requirements for LongTerm Care Facilities. Federal register. 2016. https://www.federalregister gov/documents/2016/10/04/2016-23503/medicare-and-medicaid-progr ams-reform-of-requirements-for-long-term-care-facilities. Accessed 8 Nov 2016

14. The Core Elements of Antibiotic Stewardship for Nursing Homes | Nursing Homes and Assisted Living (LTC) | CDC. http://www.cdc.gov/longt ermcare/prevention/antibiotic-stewardship.html. Accessed 25 June 2016

15. Kabbani S, Palms DL, Bartoces M, Marek J, Stone ND, Hicks LA, et al. Potential utility of pharmacy data to measure antibiotic use in nursing homes. Infect Control Hosp Epidemiol. 2019;40:819-20.

16. Song S, Wilson BM, Bej T, Gravenstein S, Carter RR, Marek J, et al. Antibiotic use among residents receiving skilled nursing care in 29 U.S. nursing homes. J Am Geriatr Soc. 2021. https://doi.org/10.1111/jgs.16856.
17. Long-Term Care: Facts on Care in the US - LTCFocus.org. http://www.ltcfo cus.org/. Accessed 25 Nov 2019.

18. Medicare.gov Nursing Home Compare. https://www.medicare.gov/nursi nghomecompare/search.html. Accessed 27 June 2014.

19. Mylotte JM. Antimicrobial stewardship in long-term care: metrics and risk adjustment. J Am Med Dir Assoc. 2016;17(672):e13-18.

20. Core Elements of Hospital Antibiotic Stewardship Programs | Get Smart for Healthcare | CDC. http://www.cdc.gov/getsmart/healthcare/imple mentation/core-elements.html. Accessed 30 Aug 2015.

21. Berrington A. Antimicrobial prescribing in hospitals: be careful what you measure. J Antimicrob Chemother. 2010;65:163-8.

22. Jump RLP, Gaur S, Katz MJ, Crnich CJ, Dumyati G, Ashraf MS, et al. Template for an antibiotic stewardship policy for post-acute and long-term care settings. J Am Med Dir Assoc. 2017;18:913-20.

23. Gerber JS, Hersh AL, Kronman MP, Newland JG, Ross RK, Metjian TA. Development and application of an antibiotic spectrum index for benchmarking antibiotic selection patterns across hospitals. Infect Control Hosp Epidemiol. 2017;38:993-7.

24. Shiny. https://shiny.rstudio.com/. Accessed 10 Apr 2021.

25. Cohen CC, Dick AW, Agarwal M, Gracner T, Mitchell S, Stone PW. Trends in antibiotics use among long-term US nursing-home residents. Infect Control Hosp Epidemiol. 2021;42(3):311-7.

26. Mylotte JM, Keagle J. Benchmarks for antibiotic use and cost in long-term care. J Am Geriatr Soc. 2005;53:1117-22.

27. Dellit TH, Owens RC, McGowan JE, Gerding DN, Weinstein RA, Burke $J P$, et al. Infectious diseases society of America and the society for healthcare epidemiology of America guidelines for developing an institutional program to enhance antimicrobial stewardship. Clin Infect Dis. 2007:44:159-77.

28. Times TNY. One-third of U.S. Coronavirus deaths are linked to nursing homes. The New York Times. 2020. https://www.nytimes.com/interactive/ 2020/us/coronavirus-nursing-homes.html. Accessed 11 Apr 2021.

29. Schirmer PL, Mercier RC, Ryono RA, Nguyen N, Lucero CA, Oda G, et al. Comparative assessment of antimicrobial usage measures in the department of veterans affairs. Infect Control Hosp Epidemiol. 2012;33:409-11.

30. Szczepura A, Wild D, Nelson S. Medication administration errors for older people in long-term residential care. BMC Geriatr. 2011;11:82.

31. Davidson HE, Jump RLP. Challenges in tracking and reporting antibiotic use in long-term care. J Am Med Dir Assoc. 2020;21 (9):1191-6.

32. Long-term Care Facilities | NHSN | CDC. http://www.cdc.gov/nhsn/LTC/ index.html. Accessed 17 Jul 2016.

\section{Publisher's Note}

Springer Nature remains neutral with regard to jurisdictional claims in published maps and institutional affiliations.
Ready to submit your research? Choose BMC and benefit from:

- fast, convenient online submission

- thorough peer review by experienced researchers in your field

- rapid publication on acceptance

- support for research data, including large and complex data types

- gold Open Access which fosters wider collaboration and increased citations

- maximum visibility for your research: over $100 \mathrm{M}$ website views per year

At BMC, research is always in progress.

Learn more biomedcentral.com/submissions 